\title{
Cyclic Changes of Lymphatic and Venous Vessels in Human Endometrium
}

\author{
Tatsuo Tomita ${ }^{1,2,3^{*}}$, Kuni Mah ${ }^{3}$ \\ ${ }^{1}$ Departments of Integrative Biosciences, Oregon Health and Science University, Portland, Oregon, USA \\ ${ }^{2}$ Departments of Pathology, Oregon Health and Science University, Portland, Oregon, USA \\ ${ }^{3}$ Oregon National Primate Center, Oregon Health and Science University, Portland, Oregon, USA \\ Email: *tomitat@ohsu.edu
}

Received 28 July 2014; revised 28 August 2014; accepted 18 September 2014

Copyright (C) 2014 by authors and Scientific Research Publishing Inc.

This work is licensed under the Creative Commons Attribution International License (CC BY). http://creativecommons.org/licenses/by/4.0/

(c) (i) Open Access

\section{Abstract}

Context: Cyclic changes of endometrial arteries are well established but possible cyclic changes of lymphatic and venous vessels have not been fully documented. There are no published morphological reports to support cyclic changes of endometrial lymphatic and venous vessels. Objective: Using cryosections of human endometrium, this study aimed to unveil possible cyclic changes of lymphatic and venous vessels. We previously reported cyclic changes of lymphatic vessels in human endometrium using D2-40. Design: A total of 16 cases representing menstrual, proliferative and mid and late secretary phase were studied. For Immunocytochemical staining, lymphatic vessel endothelial hyaluronan receptor 1 and von Willebr and factor were used for lymphatic and venous vessels, respectively. We used polyclonal LYVE-1 in this study, which revealed more lymphatic vessels than using D2-40. Results: Residual lymphatic and venous vessels were present in menstrual basalis. In Day 5 - 9 endometrium, there were sparse lymphatic vessels but were numerous growing venous vessels in thin proliferating functionalis. In Day 14 - 22 endometrium, there were scattered lymphatic vessels and numerous venous vessels in functionalis. In Day 25 26 endometrium, there were many dilated lymphatic vessels and numerous dilated, disintegrating venous vessels in upper functionalis than lower functionalis. Conclusion: The above findings support that lymphatic vessels are sparse but venous vessels are numerous in early proliferative functionalis. Lymphatic vessels grow from basalis to thin functionalis. In premenstrual phase, lymphatic vessels proliferate from lower to upper functionalis, and both lymphatic and venous vessels disintegrate for shedding by this immunocytochemical study using lymphatic and venous markers. Thus, all lymphatic, venous and arterial vessels undergo menstrual cyclic changes and shed for menstruation.

\section{Keywords}

Factor-8, Human Endometrium, Lymphatic Vessels, LYVE-1, Venous Vessels

\footnotetext{
${ }^{*}$ Corresponding author.
} 


\section{Introduction}

The cyclic changes of endometrial arteries have been well established but similar changes of venous and lymphatic vessels have not been documented [1]-[3]. Using cryosections of human endometrium of menstrual, proliferative and secretary phase, this study aimed to unveil possible cyclic changes of venous and lymphatic vessels in the menstrual period, early proliferative, mid-secretary and late-secretary phase. For immunostaining, lymphatic vessel endotheliumhyarulonan receptor 1 (LYVE-1) was used for lymphatic vessels and von Willebr and factor (F-8) was used for venous vessels, respectively [4] [5]. Polyclonal LYVE-1 revealed more lymphatic vessels than using monoclonal D2-40 (Unpublished data). Information on the endometrial lymphatic vessels is sketchy and unsettled: some authors claimed that lymphatic vessels were absent in the human endometrium [6] [7] but more investigators agree that some lymphatic vessels are present in human endometrium: one group reported that lymphatic vessels were present in functionalis in $62 \%$ of the samples [8], and another group reported that endometrial lymphatic vessels were observed in basalis alone using a lymphatic vessel immunocytochemical marker [9]. Rogers and his associates studied lymphatic vessel density with formalin-fixed and paraffinembedded human endometrium using D2-40 as a lymphatic marker: lymphatic vessels were identified in functionalis at a reduced density than basalis and myometrium [10] [11]. Lymphatic vessels in the functionalis were small and sparsely distributed whereas lymphatic vessels in the basalis were larger and often closely associated with spiral arteries/arterioles [10]-[12]. There are numerous endometrial venous vessels in all phases of menstrual cycle but clear cyclic changes of venous vessels in the endometrium have not been documented by immunocytochemical studies. Morphogenesis of lymphatic and venous vessels is associated with endometrial arteries, and lymphatic and venous vessels may be closely coordinated with cyclic changes of spiral arteries of the functionalis. And this coordinating morphogenesis is the main objective of this study.

\section{Materials and Methods}

Endometrial tissue was collected from 16 adult Caucasian women (age range-37 to 45 years) undergoing elective hysterectomy. Written informed consent was provided by all subjects and ethical approval for tissue collection granted by the Lothian Research Ethics Committee as described before [13]-[15]. All women reported regular menstrual cycles (25 - 35 days) and had not received exogenous hormones or used an intrauterine device in the 3 months prior to inclusion in this study. After the uterus had been removed, a wedge of tissue from the endometrial surface to the myometrium including the full thickness of endometrium of corpus uteri with the contiguous myometrium was taken as described before at an average size of $1 \times 1 \times 0.4 \mathrm{~cm}$ [16]. Fresh wedge tissues were embedded in OCT matrix (Fisher Scientific, Pittsburgh, PA), and were frozen in liquid propane in the liquid nitrogen bath as described before [13]-[15] and cryosectioned at 5 - 7 microns. Cryosections were mounted on Super Frost Plus slides (Fisher Scientific), microwave-irradiated on ice for $3 \mathrm{sec}$, fixed in 2\% paraformaldehyde in phosphate buffer at $\mathrm{pH} 7.4$ for 10 to $15 \mathrm{~min}$ at room temperature, and immersed twice for 2 min each time in $85 \%$ ethanol [17]. To inhibit endogenous peroxidase activity, sections were incubated with a solution containing glucose oxidase $(1 \mathrm{U} / \mathrm{ml})$ and sodium azide $(10 \mathrm{mmol} / \mathrm{ml})$ in $\mathrm{PBS}$ for $45 \mathrm{~min}$ at $25^{\circ} \mathrm{C}$ [17] [18]. Sections were incubated with blocking serum for $20 \mathrm{~min}$. Then sections were incubated with goat antihuman LYVE-1 (R and D System, Minneapolis, MN) at 1:600 dilution for lymphatic vessels or with rabbit antihuman F-8 (Dako System, Carpenteria, CA) at 1:800 dilution for venous vessels, respectively, overnight at $4^{\circ} \mathrm{C}$ [18]. After rinsing and immersion in blocking serum again, sections were incubated with second antibody (1:200 dilution) for $30 \mathrm{~min}$ at room temperature. Final visualization was achieved with the ABC kit (Vector Laboratories, Burlingame, CA) and 0.025\% diaminobenzidine tetrahydrochloride (Dojindo Molecular Technology, Rockville, MD) in Tris-buffer $\mathrm{pH}$ 7.6, $0.03 \% \mathrm{H}_{2} \mathrm{O}_{2}$ (Fisher Scientific) to induce a brown coloration. Tissue sections were then lightly counterstained with hematoxylin to facilitate identification of cellular components. Since lymphatic vessels were mostly linear with narrow lumens $(<20 \mu \mathrm{m})$, morphometric analysis of lymphatic vessels was performed for measuring the length of lymphatic vessels in five randomly selected $10 \times 10=\times 100$ fields in $\mu \mathrm{m}$ using a linear $1 \mathrm{~cm}$ scale mounted in the $10 \times$ eye piece for each case as described before [16] for the adjacent myometrium, basalis and functionalis, respectively, excluding small lymphatic vessels $(<30 \mu \mathrm{m})$ since the small lymphatic vessels were often indistinguishable from the fibrous cytoplasm of activated macrophages, which were also positive for LYVE-1 [16]-[18]. Venous vessels are more dilated than arteries and lymphatic vessels and F-8 positive venous vessels $>30 \mu \mathrm{m}$ were included in this study for the venous vessels. From the mid-proliferative to late-secretary phase, the functionalis was divided approximately into an upper one-half, a superficial 
layer, the compacta, consisting of densely packed stromal cells around the straight necks of glands, and into a lower one-half layer as spongiosa, a thick, spongy layer containing the tortuous bodies of the glands [1] [2]. The functionalis was studied for the lower functionalis during early-proliferative phase, and for both upper and lower functionalis from mid-proliferating to late-secretary phase. The total length of lymphatic and venous vessels was cumulatively measured in the microscopic slides, and the mean, SE and p values were calculated together with the total numbers of lymphatic vessels for early-proliferative phase, early-secretary phase, late-secretary phase and menstrual period (Table 1).

Table 1. Venous and lymphatic vessels in endometrium and myometrium.

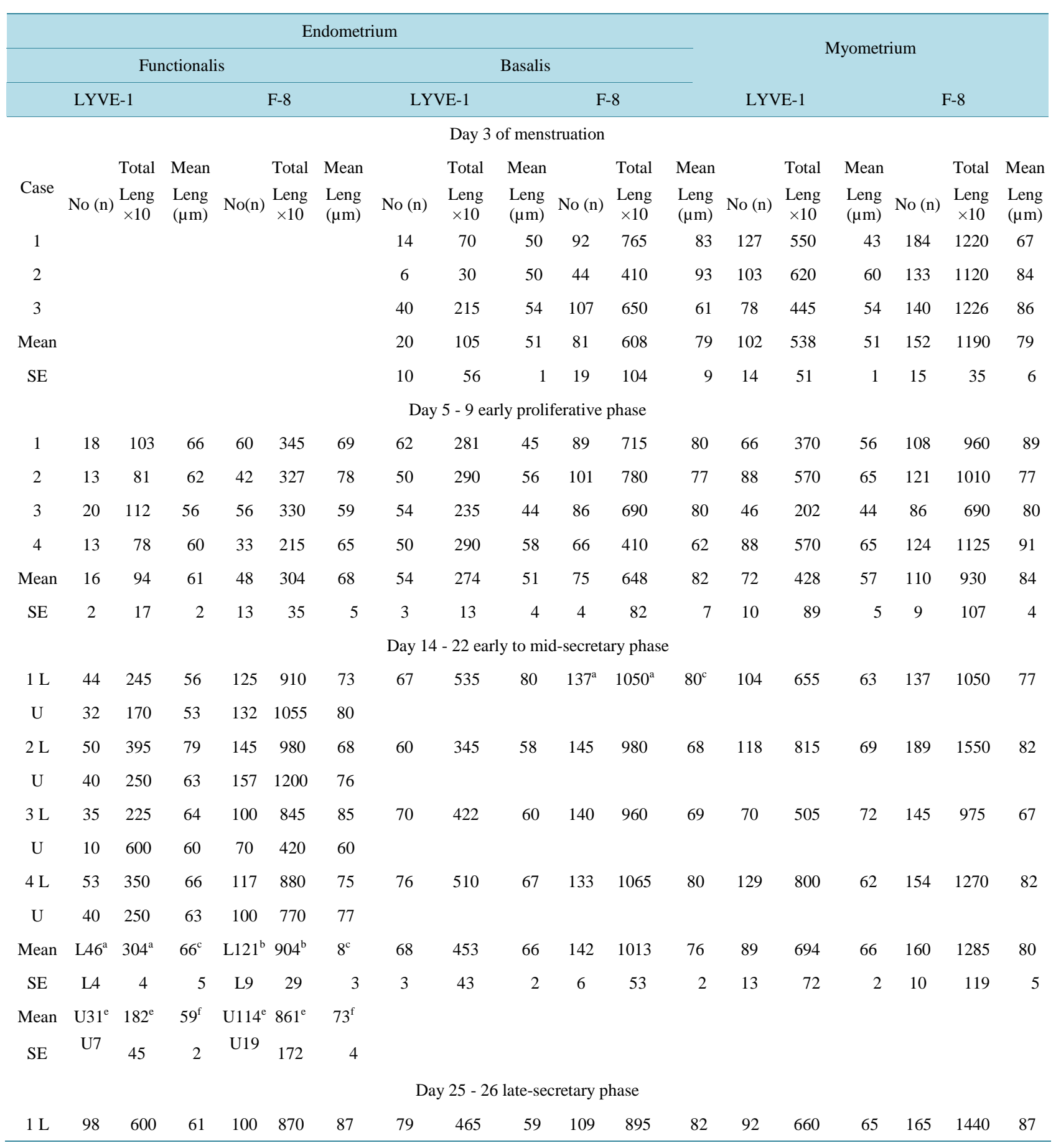




\begin{tabular}{|c|c|c|c|c|c|c|c|c|c|c|c|c|c|c|c|c|c|c|}
\hline \multicolumn{19}{|c|}{ Continued } \\
\hline $\mathrm{U}$ & 70 & 430 & 61 & 95 & 750 & 79 & & & & & & & & & & & & \\
\hline $2 \mathrm{~L}$ & 108 & 710 & 66 & 123 & 945 & 77 & 96 & 585 & 61 & 133 & 1230 & 92 & 214 & 1285 & 60 & 190 & 1590 & 84 \\
\hline $\mathrm{U}$ & 98 & 620 & 63 & 122 & 870 & 71 & & & & & & & & & & & & \\
\hline $3 \mathrm{~L}$ & 76 & 455 & 66 & 154 & 1250 & 81 & 86 & 490 & 57 & 170 & 1200 & 71 & 145 & 840 & 58 & 222 & 1630 & 73 \\
\hline $\mathrm{U}$ & 65 & 390 & 60 & 107 & 765 & 71 & & & & & & & & & & & & \\
\hline $4 \mathrm{~L}$ & 93 & 555 & 60 & 122 & 965 & 79 & 107 & 670 & 63 & 139 & 1225 & 88 & 163 & 1035 & 63 & 160 & 1630 & 102 \\
\hline $\mathrm{U}$ & 78 & 450 & 58 & 84 & 685 & 82 & & & & & & & & & & & & \\
\hline $5 \mathrm{~L}$ & 74 & 400 & 54 & 108 & 735 & 68 & 61 & 327 & 54 & 119 & 742 & 62 & 65 & 350 & 54 & 147 & 1197 & 81 \\
\hline $\mathrm{U}$ & 52 & 290 & 56 & 82 & 485 & 59 & & & & & & & & & & & & \\
\hline L Mean & 90 & $544^{\mathrm{a}}$ & $60^{c}$ & $121^{\mathrm{a}}$ & $953^{\mathrm{a}}$ & $78^{c}$ & 86 & 506 & 59 & 134 & 1140 & 79 & 136 & 822 & 60 & 177 & 1497 & 85 \\
\hline L SEM & 7 & 54 & 2 & 9 & 84 & 3 & 8 & 58 & 2 & 10 & 153 & 6 & 26 & 163 & 2 & 13 & 83 & 5 \\
\hline U Mean & $72^{\mathrm{e}}$ & $436^{e}$ & $59^{f}$ & $98^{\mathrm{e}}$ & $711^{\mathrm{e}}$ & $72^{f}$ & & & & & & & & & & & & \\
\hline U SEM & 8 & 54 & 2 & 7 & 64 & 4 & & & & & & & & & & & & \\
\hline
\end{tabular}

P values between lower functionalis and basalis: $\mathrm{a}<0.005, \mathrm{~b}<0.01$, c: non-significant. P values between upper functionalis and lower functionalis: $\mathrm{d}<0.001, \mathrm{e}<$ 0.05 , f: non-significant.

\section{Results}

In Day 3 endometrium, there were some lymphatic and numerous venous vessels in basalis as compared to more than five times lymphatic and about twice more, large venous vessels in the myometrium, respectively (Figure 1(A) and Figure 1(B), Table 1). The sizes of non-cycling lymphatic and venous vessels in the basalis were about the same of those in the myometrium (Table 1). In Day 5 - 9 basalis, there were practically no lymphatic vessels but were numerous elongated venous vesselsin the functionalis, perpendicularly to the uterine cavity (Figure 1(D)). The numbers and total lengths of lymphatic vessels increased more than twice in the Day 5 - 9 basalis than the Day 3 basalis (Table 1). The numbers and mean lengths of lymphatic and venous vessels were about the same for Day3 and Day 5 - 9 myometrium (Table 1). In Day 14 - 22 endometrium, there were a few lymphatic and numerous venous vessels, longitudinal to the endometrial cavity in the functionalis as compared to numerous large lymphatic and venous vessels in the basalis and most numerous horizontal venous vessels in the myometrium (Figures 2(A)-(D)). The lower functionalis revealed more than 1.5 times numbers and more total lengths of lymphatic vessels compared to those of the upper functionalis but the mean lengths of lymphatic vessels were about the same for the upper and lower functionalis (Table 1). The total numbers, total lengths and mean lengths of venous vessels in the Day 14 - 22 functionalis were about the same for the upper and lower functionalis (Table 1). The total numbers and total lengths of lymphatic vessels in the functionalis were more than three times those of Day 5 - 9 functionalis (Table 1). In Day 25 - 26 endometrium, upper functionalis revealed less longitudinal lymphatic vessels than lower functionalis as compared to numerous small lymphatic vessels in basalis and more numerous, large horizontal lymphatic vessels in the basalis and myometrium (Figure 3(A) and Figure 3(B)). Compared with the Day 14 - 22 upper functionalis, which revealed more lymphatic vessels than the Day 25 - 26 upper functionalis, the Day 25 - 26 uterus revealed about 10\% more lymphatic and venous vessels for basalis and about $15 \%$ more lymphatic and venous vessels for myometrium than the corresponding Day 14 - 22 basalis and myometrium, respectively (Table 1). The radial arterial endothelium in the endometrial-myometrial junction was weaker immunostained for F-8 than venous vessel endothelium, the latter was much strongerimmunostained than the former (Figure 3(D)). Compared with the Day 25 - 26 functionalis, the Day 14 - 22 functionalis often showed longer longitudinal lymphatic vessels as the Day 14 - 22 upper functionalis revealed more total numbers and total lengths of venous vessels than those of Day 25 - 26 upper functionalis (Table 1). In the Day 25 - 26 functionalis, there were more increased larger, occasional large diluted, degenerating venous vessels with disintegrated wall, which were prominently present in the upper functionalis with similar but less dilated venous vessels were observed in the lower functionalis (Figure 4(C) and Figure 4(D)). 


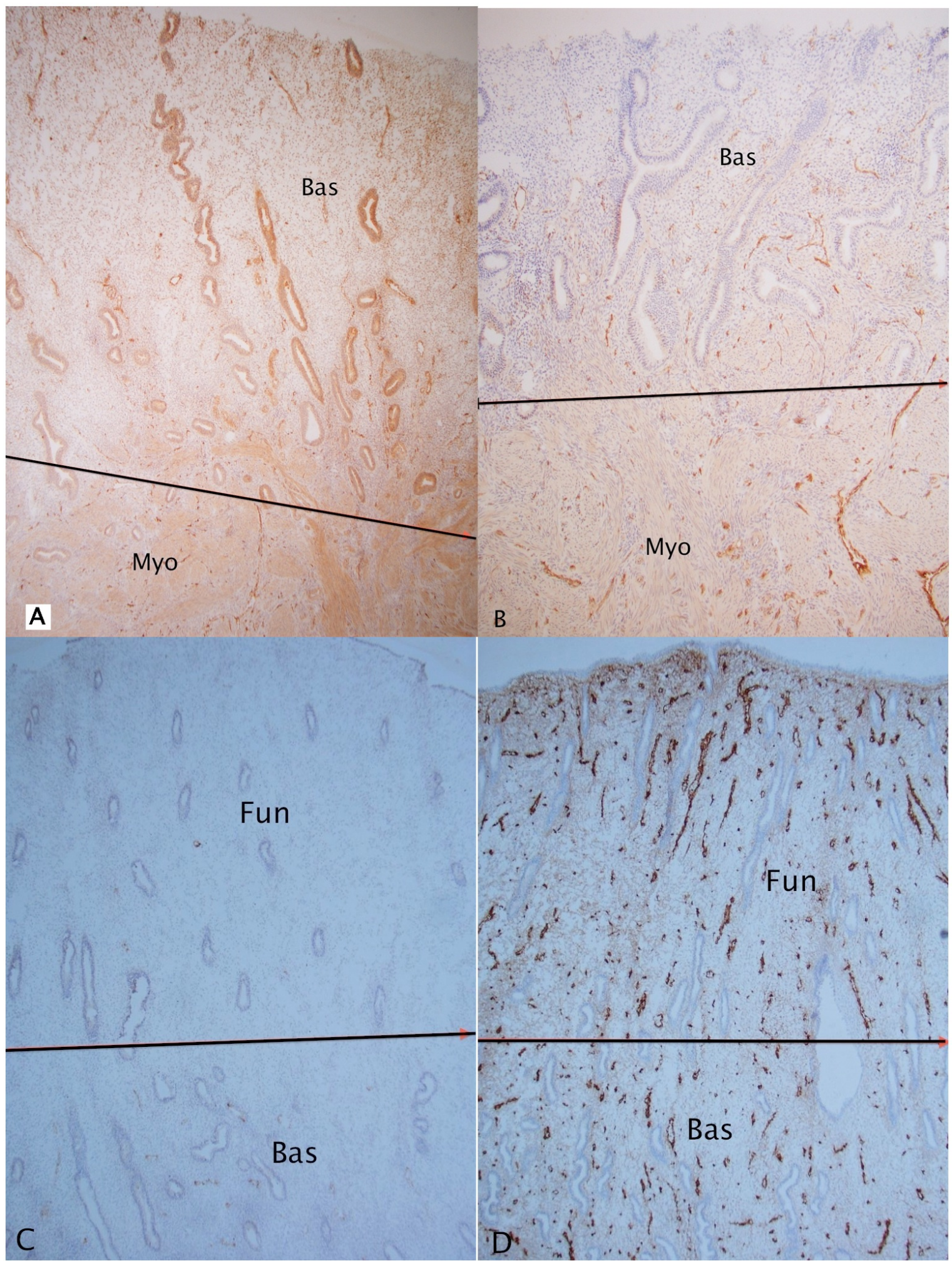

Figure 1. Day 3, Case 2 (A) and (B) and Day 5 - 9, Case 1 endometrium (C) and (D). In Day 3 endometrium (A) and (B), there were few non-cycling lymphatic vessels (A) and numerous small venous vessels in basalis (B) as compared to numerous lymphatic vessels and large numerous venous vessels in myometrium. In Day 5 - 9 endometrium, there were no lymphatic vessels (C) but numerous elongated venous vessels (D) perpendicular to the uterine cavity in functionalis whereas there were a few small lymphatic vessels and numerous short venous vessels in basalis (D). (A) and (C) LYVE-1; (B) and (D) F-8 immunostained. 


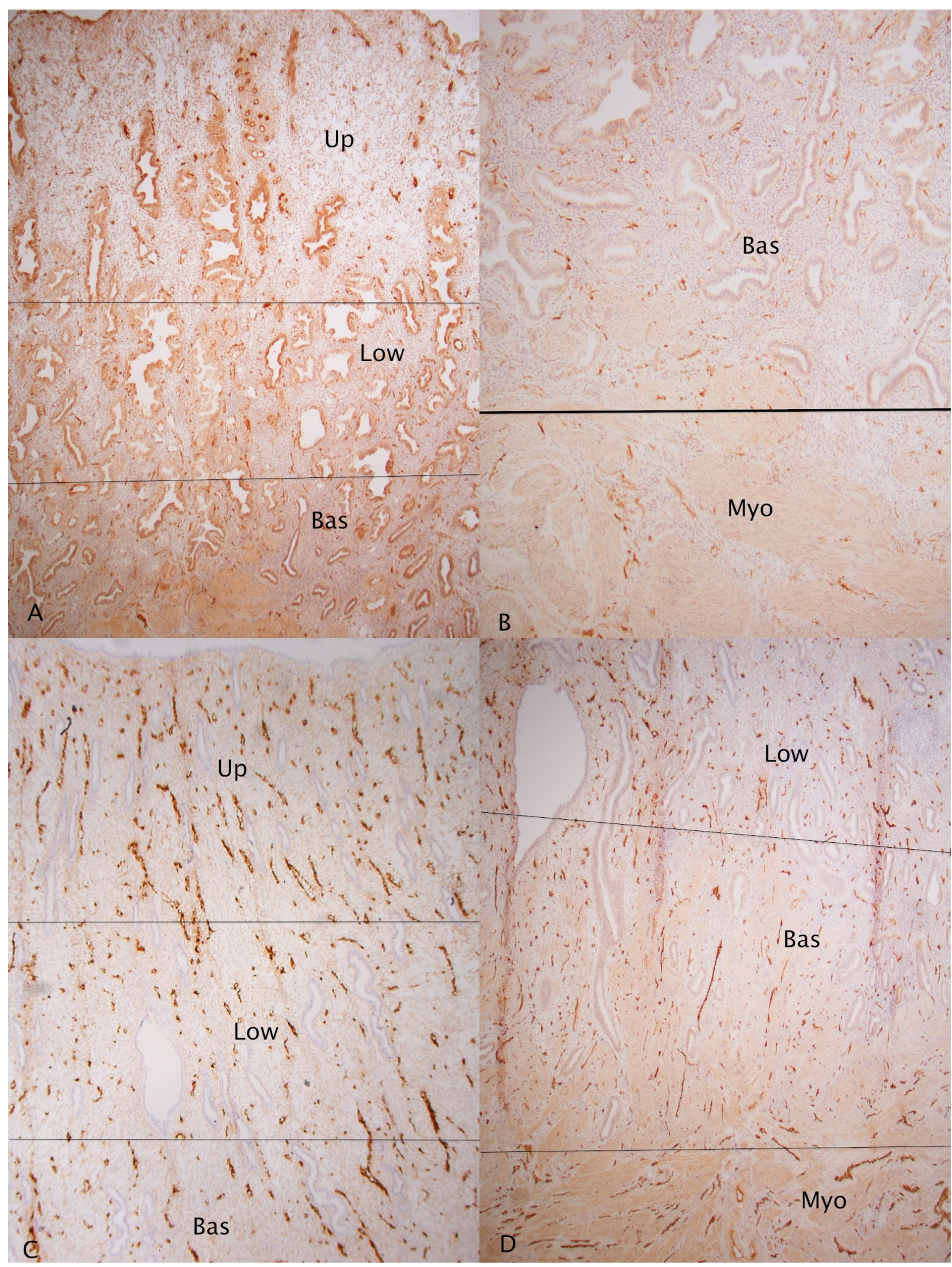

Figure 2. Day 14 - 22, Case 3 endometrium (A)-(D). There were a few scattered lymphatic vessels in upper functionalis and some lymphatic vessels in lower functionalis and basalis as compared to numerous large lymphatic vessels in myometrium (A) and (B). There were numerous larger venous vessels, perpendicular to the uterine cavity in both upper and lower functionalis and basalis whereas there were numerous venous vessels both perpendicular and transversely located in myometrium (C) and (D). (A) and (B) LYVE-1; (C) and (D) F-8 immunostained. 


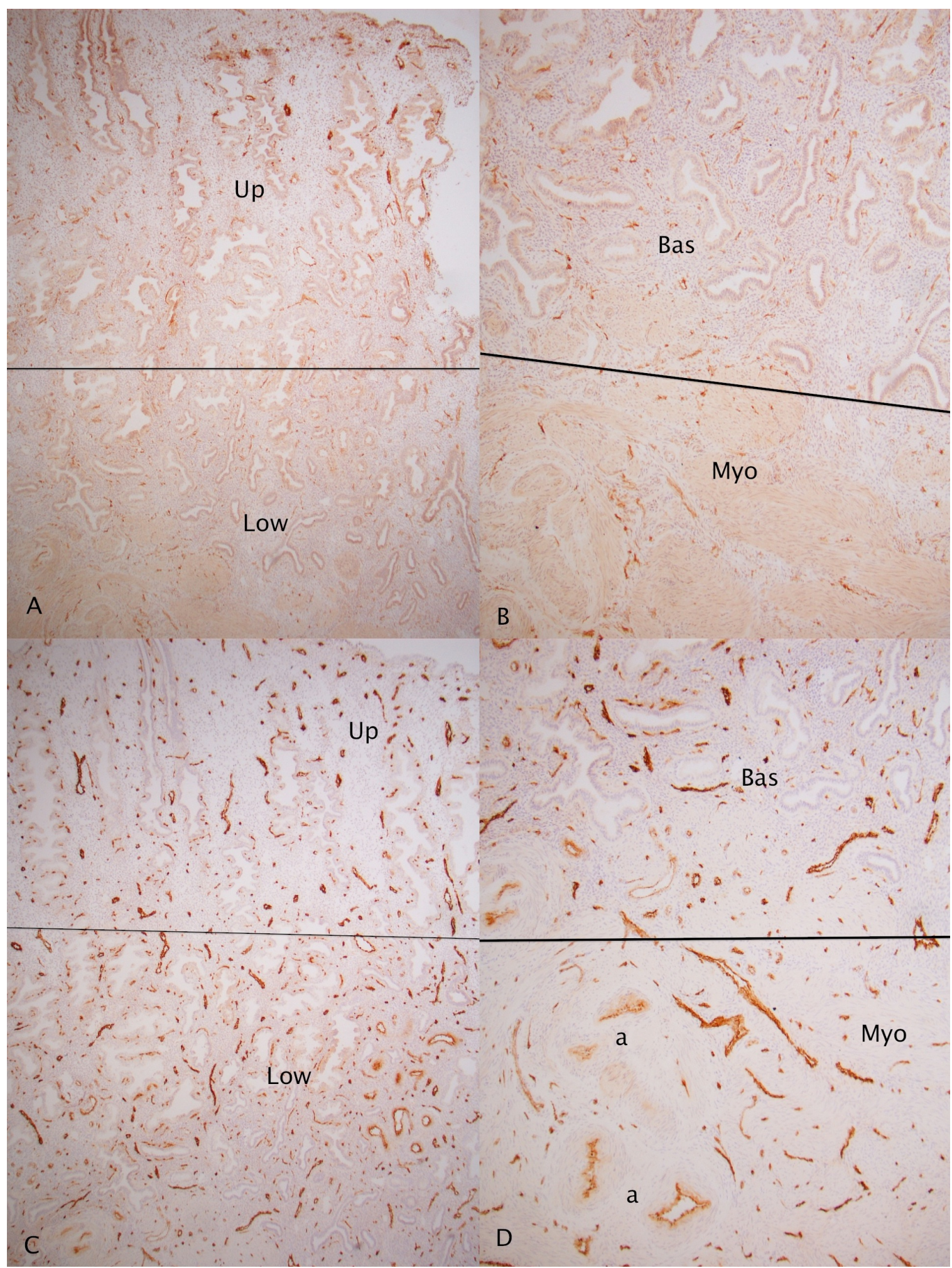

Figure 3. Day 25 endometrium, Case 5 (A)-(D). There were several perpendicular lymphatic vessels in upper functionalis as compared to numerous small lymphatic vessels in lower functionalis and basalis (A) and (B). Myometrium contained numerous both perpendicular and transverse lymphatic vessels (B). There were many smaller venous vessels perpendicular to the uterine cavity in upper functionalis as compared to larger venous vessels in lower functionalis and basalis (C) and (D). a: Radial arteries in the myometrial-endometrial junction. (A) and (B) LYVE-1; (C) and (D) F-8 immunostained. 


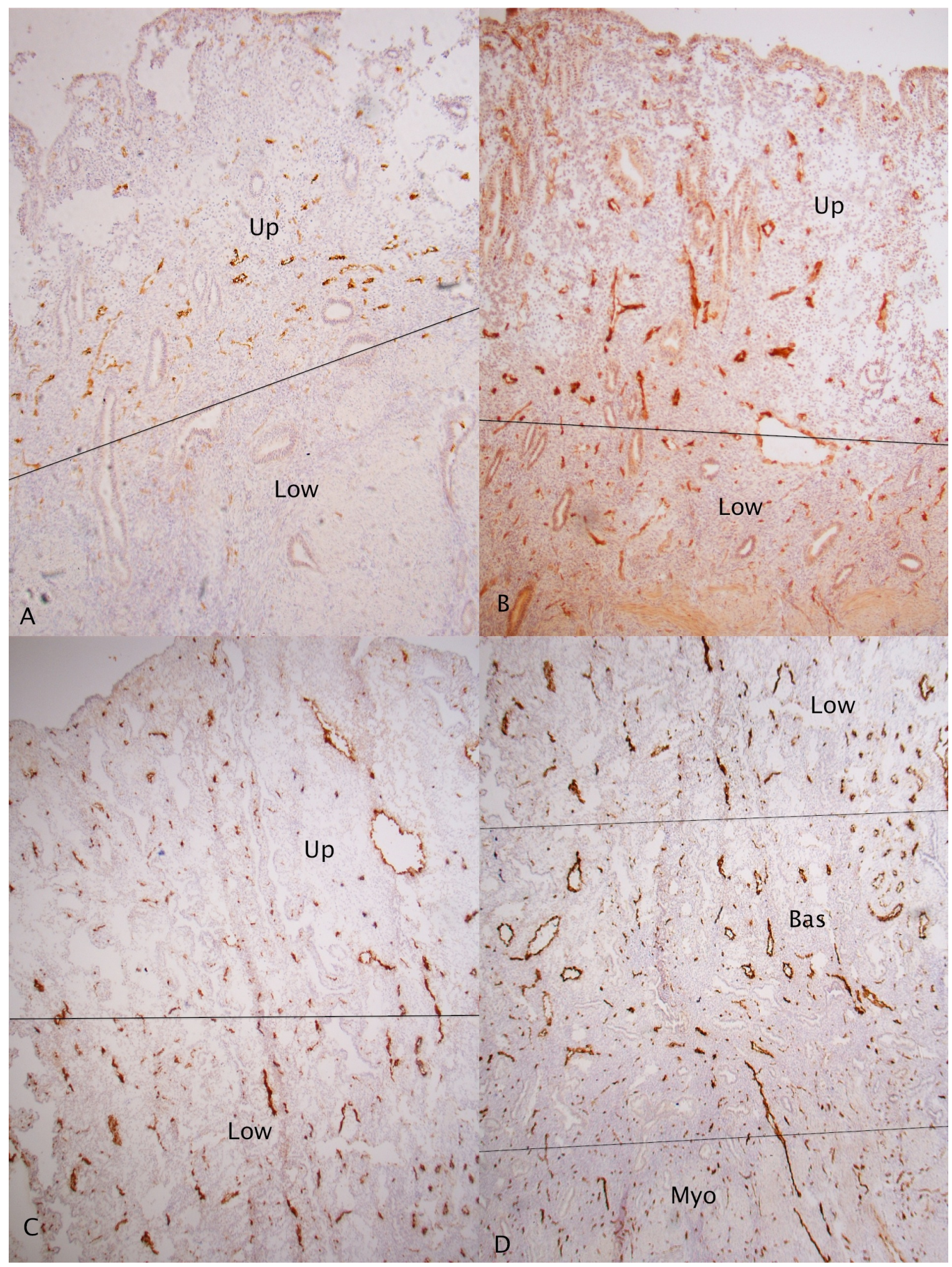

Figure 4. Day 26 endometrium Case 2 (A)-(D). There were more increased perpendicular dilated larger lymphatic vessels in upper functionalis as compared to smaller lymphatic vessels in lower functionalis (A) and (B). There were less venous vessels containing markedly dilated venous lumens compared to the Day 14 - 22 functionalis, the latter contained intact, more numerous elongated perpendicular venous vessels. There were similar but less dilated venous vessels in lower functionalis and basalis (C) and (D). (A) and (B) LYVE-1; (C) and (D) F-8 immunostained. 


\section{Discussion}

The cyclic changes of endometrial arteries of the human endometrium are well established: a gradual increase in arborization of coiling of spiral arteries during proliferative phase and the spiral growths parallel the gradual increase in length and coiling of endometrial glands in post-ovulatory period [2]. Blood to the endometrium is supplied through the radial arteries, which arise from the arcuate arteries in the myometrium. After passing through the myometrial-endometrial junction, radial arteries split into the smaller basal arteries that supply the basalis and the spiral arteries, the latter continue to supply the functionalis. The spiral arteries have distinctive coiled appearance with more pronounced coiling during the secretory phase [10]. During the menstrual period, a collapse of the arterial and glandular systems predominates in the functionalis whereas both arteries and endometrial glands are thought to remain unchanged in the basalis throughout the menstrual cycle [2]. Spiral arteries proceed to form capillary plexuses and arteriovenous anastomoses: the former subsequently form venous lakes whereas the latter merge directly to the venous vessels [10] [19]. Information on the cyclic changes of human endometrial lymphatic and venous vessels is much limited to date [6]-[9]. The presence of endometrial lymphatics is unsettled: Some authors claimed no lymphatics in the human endometrium [6] [7]. Using LYVE-1 with nonhuman primate endometrium, Red-Horse et al. reported no lymphatic vessels in the endometrium but pregnancy rapidly induced lymphatic vessels in the decidual parts of the uterus [7]. By studying 15 surgically resected uteri of normal endometrium of various phases of menstrual cycle, Koukourakis et al. detected no LYVE-1 positive lymphatic vessels in endometrium although a dense CD 31 positive vascular network was detected [6]. But more reports agreed that lymphatic vessels are present in the human endometrium: two studies reported lymphatic vessels in the functionalis of human endometrium in $62 \%$ of samples or with restricted distribution in the functionalis relative to the basalis [8] whereas another study identified endometrial lymphatic vessels in the basalis alone [9]. We had initially used the routinely formalin-fixed and paraffin-embedded human uterine tissues using LYVE-1 and D2-40, which revealed sporadic and sparse lymphatic vessels in the functionalis but we could not detect cyclic changes of lymphatic vessels in the functionalis. The recent studies using formalin-fixed and paraffin-embedded tissues identified sparse lymphatic vessels in all phases of functionalis [11] [12]. For immunostaining lymphatic vessels, LYVE-1 and D2-40 have been widely used: LYVE-1, a transmembrane receptor that binds to glucosaminoglycan hyaluronan [20] has provided a tool to accurately characterize lymphatic distribution in wide range of tissues [20]-[22]. D2-40, a novel monoclonal antibody to O-linked sialoglycoprotein that reacts with lymphatic endothelium has also been widely used for immunostaining lymphatic vessels [23] [24] and does not immunostain endothelial capillaries, arteries or veins [23]-[25]. Polyclonal LYVE-1 tended to immunostain more lymphatic vessels than monoclonal D2-40 in our experience (Unpublished data). D2-40 required no less than 1:100 dilution of the antibody for both cryosections and formalin-fixed and paraffin-embedded tissue sections for immunostaining lymphatic vessels (unpublished data). In this study, cryosections needed only a fraction of the antibody at 1:600 dilution for immunostaining lymphatic vessels using goat antihuman LYVE-1 and also lesser antibody at 1:800 diluted rabbit antihuman F-8 for immunostaining venous vessels [16] [18]. In contrast, the routinely processed paraffin sections required no less than 1:100 diluted antibody solution for immunostaining lymphatic and venous vessels, respectively [4] [5]. Thus, it appears that the routinely formalin-fixed and paraffin-embedded sections apparently lost the antigenicity for LYVE-1 and F-8 through tissue preparation compared to the cryosections. Using monoclonal D2-40, Rogers and his associates extensively studied angiogenesis and lymphangiogenesis with the routinely processed paraffin-embedded human endometrium by measuring lymphatic vessel density (LVD): no significant difference between proliferative and secretary LVD within the functionalis (proliferative $16.7 \pm 2.6 \mathrm{~mm}^{2}$ vs secretary $16.2 \pm$ $2.6 \mathrm{~mm}^{2}$ ), basalis (proliferative $73.1 \pm 3.7$ vessels $/ \mathrm{mm}^{2}$ vs secretory $79.1 \pm 7.5 \mathrm{~mm}^{2}$ ) and myometrium (proliferative $63.4 \pm 2.7$ vessels $\mathrm{mm}^{2}$ vs secretary $60.3 \pm 2.6$ vessels $\mathrm{mm}^{2}$ ) [11]. The majority of D2-40 positive lymphatic vessels were positive for CD31 but not for F-8 [11]. By comparing CD 31 with D2-40 immunostaining, the authors had estimated that $13 \%$ of the vessels profiles in functionalis, $43 \%$ in basalis and $28 \%$ in myometrium were lymphatic vessels [11]. They concluded that the LVD of functionalis was significantly reduced compared with basalis and myometrium across the cycle $(p=0.001)$ [11]. They further claimed that basalis contained 4 - 5 times more lymphatic vessels than myometrium and there was a reduced or no lymphatic vessel density in the functionalis relative to the basalis [11] [12]. Compared with the lymphatic vessel density study by Rogers et al. [11], our cryosection immunostaining revealed sparse lymphatic vessels in the functionalis of the 
early-proliferative phase and increasing lymphatic vessels in early-secretary to late-secretary phase functionalis (Figures 2-4, Table 1). Thus, lymphatic vessel immunstaining with cryosections appears to reveal more lymphatic vessels than the routine paraffin sections as reported before (Table 1). Lymphangiogenesis is rather loosely associated with angiogenesis except for those located in the periarcuate arterial stroma in the myometrium (Figure 3). The arcuate arteries in the myometrium are bigger than the radial arteries in the myometriumendometrial junction, and the latter are bigger than spiral arterioles in the functionalis [26]. Thus, more lymphatic vessels are present in the myometrium associated with arcuate arteries than the functionalis, in the latter lymphatic vessels are not associated with spiral arterioles by sporadic distribution in the upper functionalis (Figure 3 and Figure 4). The lymphatic system plays a major role in the both tissue fluid balance and immune surveillance in the human endometrium, but the functional significance of sparse endometrial lymphatics in the functionalis remains speculative today [26]. The sparse lymphatic drainage of the functionalis does provide a functional explanation for edema that has long been recognized as a histological feature of the functionalis at the specific stages of the menstrual cycle [11] [26]. It appears that dilated lymphatic vessels in the functionalis from the late-secretary phase are unique with a special function such as both absorption and release of edematous functionalis stroma. During proliferative phase when functionalis grows under estradiol control, lymphatic vessels are not as abundantly present as venous vessels in the functionalis, and lymphatic vessels are therefore not under estradiol control. Lymphatic vessels from Day 25 - 26 are sporadically distributed in the functionalis and are not continuous to the non-cycling horizontal lymphatic vessels in the basalis. Thus, there is no morphological evidence to support that the longitudinal lymphatic vessels in the Day 25 - 26 upper functionalis drain into the horizontal non-cycling lymphatic vessels in the basalis (Figure 1 and Figure 4). Further, longitudinal lymphatic vessels from the Day 25 - 26 functionalis abruptly taper off, and this finding supports that these longitudinal lymphatic vessels do not drain into the lymphatic vessels in the basalis. Maintaining fluid homeostasis is a key function of lymphatic vessels. Excess protein-rich fluid is removed from tissue via lymphatic vessels for return to the blood circulation [26]. Furthermore, lymphatic vessels have key roles in immune surveillance and transporting both soluble antigens and antigen presenting cells from peripheral tissues to the lymph nodes [10] [11] [26] [27]. During proliferative phase, endometrial blood flow rises as there is a significant correlation between unopposed circulating estradiol levels and rate of endometrial blood flow to reach a peak just before ovulation [28]. There is a significant positive correlation between unopposed circulating estradiol levels and rate of endometrial blood flow [28]. When progesterone levels are elevated after ovulation, the correlation between estradiol and blood flow is lost [28]. The increasing venous vessels are present in the functionalis from Day 14 22 to Day 25 - 26 endometrium, with increasing larger longitudinal venous vessels toward the uterine cavity, which accommodates the increasing blood flow (Figure 3). In the late-secretory phase, when estradiol and progesterone levels decline, blood flow in the functionalis declines. Dilated lymphatic and venous vessels characteristically appear at the premenstrual phase, and this thin-walled lymphatic and venous vessels are also observed in progestin-induced break-through bleeding [26]-[29]. By functional study using mouse models, Rodgers et al. reported that menstrual bleeding commences from the wall of an arterial or capillary vessels once previously constricted spiral arteries relax and blood flow recommences, which is responsible for $70 \%$ of blood loss [26]. Some blood leaves through the capillary circulation, accounting for $5 \%$ of blood loss and reflux from venous vessels through previously formed breaks, accounting for $25 \%$ of blood loss [26]. Blood loss from a break in the endometrial vasculature during menstruation normally lasts for only 1 - 2 min before ceasing due to spiral arterial vasoconstriction [26]. Thus, venous vasculature includes venous vessels and venous lakes accounts for about 30\% of menstrual blood loss [26]. The current study provided a further morphological evidence of cyclic changes of lymphatic and venous vessels, which account for a sizable percentage of menstrual blood loss. The presence of dilated, disintegrating venous vessels in upper functionalis from the Day 25 - 26 late-secretory phase also supports the venous bleeding for menstrual bleeding (Figure 3 and Figure 4). Endometrium exposed to progestin undergoes a well characterized series of morphological changes, including pseudo-decidualization of the stroma and appearance of abnormally dilated and thin-walled endometrial vessels [26]-[29]. This change represents dilated and disintegrating lymphatic and venous vessels observed in the premenstrual upper functionalis (Figure 4). Arteries/arterioles drain into the corresponding venous system: the spiral artery/arterioles in the functionalis drain into capillary plexus which subsequently drain into venous lakes. Thus, all spinal arteries in the functionalis eventually drain into the draining venous vessels in the functionalis whereas straight arteries drain into the venous vessels in the basalis [29]-[31]. As mentioned above, all arterial, capillary plexus, venous 
and lymphatic vessels undergo menstrual cyclic changes and disintegrate for the menstrual bleeding.

\section{Acknowledgements}

We want to express our sincere thanks to Dr. Hilary OD Critchley, University of Edinburg, UK for providing the human uterine tissues and Drs. Robert Brenner and OvSlayden for allowing us to use their research laboratory at National Oregon Primate Center, Beaverton, OR. This study was supported in part by ONPRC Core grant: NIH 000163.

\section{References}

[1] Buga, G.A.B. (2007) The Normal Menstrual Cycle. In: Kruger, T.F. and Batha, M.H., Eds., Clinical Gynecology, 3rd Edition, Juta \& Co., Cape Town, 73-87.

[2] Mutter, G.L. and Frenczy, A. (2002) Anatomy and Histology of the Uterine Corpus. In: Kurman, R.J., Ed., Blaunstein's Pathology of the Female Genital Tract, 5th Edition, Springer, New York, 383-419.

[3] Lumsden, M.A., McGavigean, J. (2002) Menstruation and Menstrual Disorder. In: Gynecology, Elsevier, Philadelphia, 459-476.

[4] Tomita, T. (2007) Immunocytochemical Localization of Lymphatic and Venous Vesselsincolonic Polyps and Adenomas. Digestive Diseases and Sciences, 53, 1880-1885. http://dx.doi.org/10.1007/s10620-007-0078-9

[5] Tomita, T. (2009) LYVE-1 Immunocytochemical Staining for Gastrointestinal Carcinoids. Pathology, 41, $248-253$. http://dx.doi.org/10.1080/00313020902756253

[6] Koukoulakis, M., Giatromanolaki, A., Sivridis, E., et al. (2005) LYVE-1 Immunohistochemical Assessment of Lymphangiogenesis in Endometrial and Lung Cancer. Journal of Clinical Pathology, 58, 202-206. http://dx.doi.org/10.1136/jcp.2004.019174

[7] Red-Horse, K., Rivera, J., Shatz, A., Zhou, Y., Winn, V., et al. (2006) Cytotrophoblast Induction of Arterial Apoptosis and Lymphangiogenesis in an in Vitro Model of Human Placentation. Journal of Clinical Investigation, 116, 26432652. http://dx.doi.org/10.1172/JCI27306.

[8] Blackwell, P. and Fraser, I. (1981) Superficial Lymphatics in the Functionalis Zone of Normal Human Endometrium. Microvascular Research, 21, 142-152. http://dx.doi.org/10.1016/0026-2862(81)90027-3

[9] Uchino, S., Ishikawa, S., Okuno, M., Nakamura, Y. and Imura, A. (1987) Methods of Detection of Lymphatics and Their Changes with Oesterous Cycle. International Angiology, 6, 271-278.

[10] Garling, J.E. and Rogers, P.A.W. (2012) The Endometrial Lymphatic Vasculature: Function and Dysfunction. Reviews in Endocrine and Metabolic Disorders, 13, 265-275. http://dx.doi.org/10.1007/s11154-012-9224-6

[11] Rogers, P.A.W., Donoghue, J.F. and Girling, J.E. (2008) Endometrial Lymphangiogenesis. Placenta, 22, 48-54. http://dx.doi.org/10.1016/j.placenta.2007.09.009

[12] Rogers, P.A.W., Donoghue, J.F., Walter, L.M., et al. (2009) Endometrial Angiogenesis, Vascular Maturation and Lymphangiogenesis. Reproductive Sciences, 16, 147-151. http://dx.doi.org/10.1177/1933719108325509

[13] Brenner, R.M., Slayden, O.D., Rodgers, W.H., et al. (2003) Immunocytochemical Assessment of Mitotic Activity with an Antibody to Phosphorylated Histone H3 in the Macaque and Human Endometrium. Human Reproduction, 18, 11851193. http://dx.doi.org/10.1093/humrep/deg255

[14] Nayak, N.R., Critchley, H.O.D., Slayden, O.D., Menrad, A., Schwalisz, K., Baird, D.T. and Brenner, R.M. (2000) Progesterone Withdrawal Up-Regulates Vascular Endothelial Growth Factor Receptor Type 2 in the Superficial Zone Stroma of the Human and Macaque Endometrium. The Journal of Clinical Endocrinology and Metabolism, 85, 34433452.

[15] Slayden, O.D., Nayak, N.R., Burton, K.A., Chwalisz, K., Cameron, S.T., Critchley, H.O.D., Baird, D.T. and Brenner, R.M. (2001) Progesterone Antagonists Increase Androgen Receptor Expression in the Rhesus Macaque and Human Endometrium. The Journal of Clinical Endocrinology and Metabolism, 86, 2668-2679.

[16] Tomita, T. (2014) Cyclic Changes of Lymphaticvessels in Human Endometrium. Open Journal of Pathology, 4, 5-12. http://dx.doi.org/10.4236/ojpathology.2014.41002

[17] Slayden, O.D., Koji, T. and Brenner, R.M. (1995) Microwave Stabilization Enhances Immunocytochemical Detection of Estrogen Receptor in Frozen Sections of Macaque Oviduct. Endocrinology, 136, 4012-4021.

[18] Tomita, T., Mah, K., Cao, W.G., Brenner, R.M., et al. (2006) Cyclic Changes in Endometrial Lymphatics. Journal of the Society for Gynecologic Investigation, 13, 81A. (Abstract)

[19] Rogers, P.A. (1996) Structure and Function of Endometrial Blood Vessels. Human Reproduction, 2, 57-62. 
http://dx.doi.org/10.4236/ojpathology.2014.41002

[20] Benerg, S., Ni, J., Wang, S.X., Clasper, S., Su, J., et al. (1999) Lyve-1, a New Homologue of the CD 44 Glycoprotein, Is a Lymph Specific Receptor for Hyaluronan. The Journal of Cell Biology, 144, 789-801. http://dx.doi.org/10.1083/jcb.144.4.789

[21] Jackson, D.J. (2003) The Lymphatics Revisited: New Prospective from the Hyaluronan Receptor LYVE-1. Trends in Cardiovascular Medicine, 13, 1-7. http://dx.doi.org/10.1016/S1050-1738(02)00189-5

[22] Jackson, D.G. (2009) Immunological Features of Hyaluronan and Its Receptors in the Lymphatics. Immunological Reviews, 230, 216-231. http://dx.doi.org/10.1111/j.1600-065X.2009.00803.x

[23] Marks, A., Sutherland, D.R., Bailey, D., Iglesias, J., Law, J., et al. (1999) Characterization and Distribution of an Oncofetal Antigen (M2A Antigen) Expressed on Testicular Germ Cell Tumors. British Journal of Cancer, 80, 569-578. http://dx.doi.org/10.1038/sj.bjc.6690393

[24] Franke, F.E., Pauls, K., Marks, S., Bergmann, M., et al. (2002) Differentiation Markers of Sertoli Cells and Germ Cells in Fetal and Early Postnatal Human Testis. Anatomy and Embryology, 209, 169-177.

[25] Kahn, H.J. and Marks, A. (2002) A New Monoclonal Antibody, D2-40, for Detection of Lymphatic Invasion of Primary Tumors. Laboratory Investigation, 82, 1255-1257. http://dx.doi.org/10.1097/01.LAB.0000028824.03032.AB

[26] Rogers, P.A.W., Donoghue, J.F., Walter, L.M. and Girling, J.E. (2009) Endometrial Angiogenesis, Vascular Maturation and Lymphangiogenesis. Reproductive Sciences, 16, 147-151. http://dx.doi.org/10.1177/1933719108325509

[27] Uyeki, M. (1991) Histologic Study of Endometriosis and Examination of Lymph Drainage in and from Uterus. American Journal of Obstetrics \& Gynecology, 165, 201-209. http://dx.doi.org/10.1016/0002-9378(91)90252-M

[28] Fraser, I.S. and Peek, M.J. (1992) Effects of Exogenous Hormonesones on Endometrialcapillaries. In: Alexander, N.J. and d'Arcangues, C., Eds., Steroid Hormones and Uterine Bleeding, AAAS Press, Washington DC, 67-79.

[29] Donoghue, J.F., McGavigan, C.J., Lederman, F.L., Cann, L.M., Fu, L., et al. (2012) Dilated Thin-Walled Blood and Lymphatic Vessels in Human Endometrium: A Potential Role for VEGF-D in Progestin-Induced Break-Through Bleeding. PLos ONE, 7, e30916. http://dx.doi.org/10.1371/journal.pone.0030916

[30] Frenczy, A., Bertrand, G. and Gelfand, M.M. (1979) Proliferation Kinetics of Human Endometrium during the Normal Menstrual Cycle. American Journal of Obstetrics \& Gynecology, 133, 859-869.

[31] Ramsey, E.M. (1982) Vascular Anatomy. In: Wynn, R.M., Ed., Biology of the Uterus, 2nd Edition, Plenum Press, New York, 59-76. 
Scientific Research Publishing (SCIRP) is one of the largest Open Access journal publishers. It is currently publishing more than 200 open access, online, peer-reviewed journals covering a wide range of academic disciplines. SCIRP serves the worldwide academic communities and contributes to the progress and application of science with its publication.

Other selected journals from SCIRP are listed as below. Submit your manuscript to us via either submit@scirp.org or Online Submission Portal.
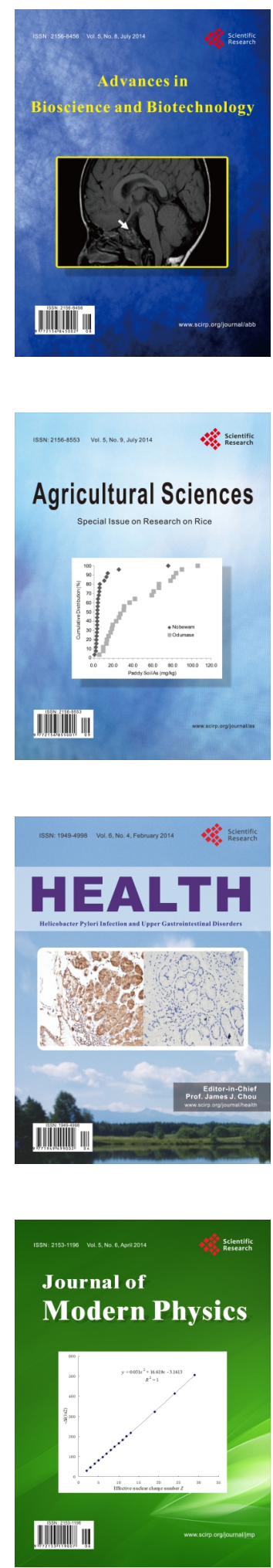
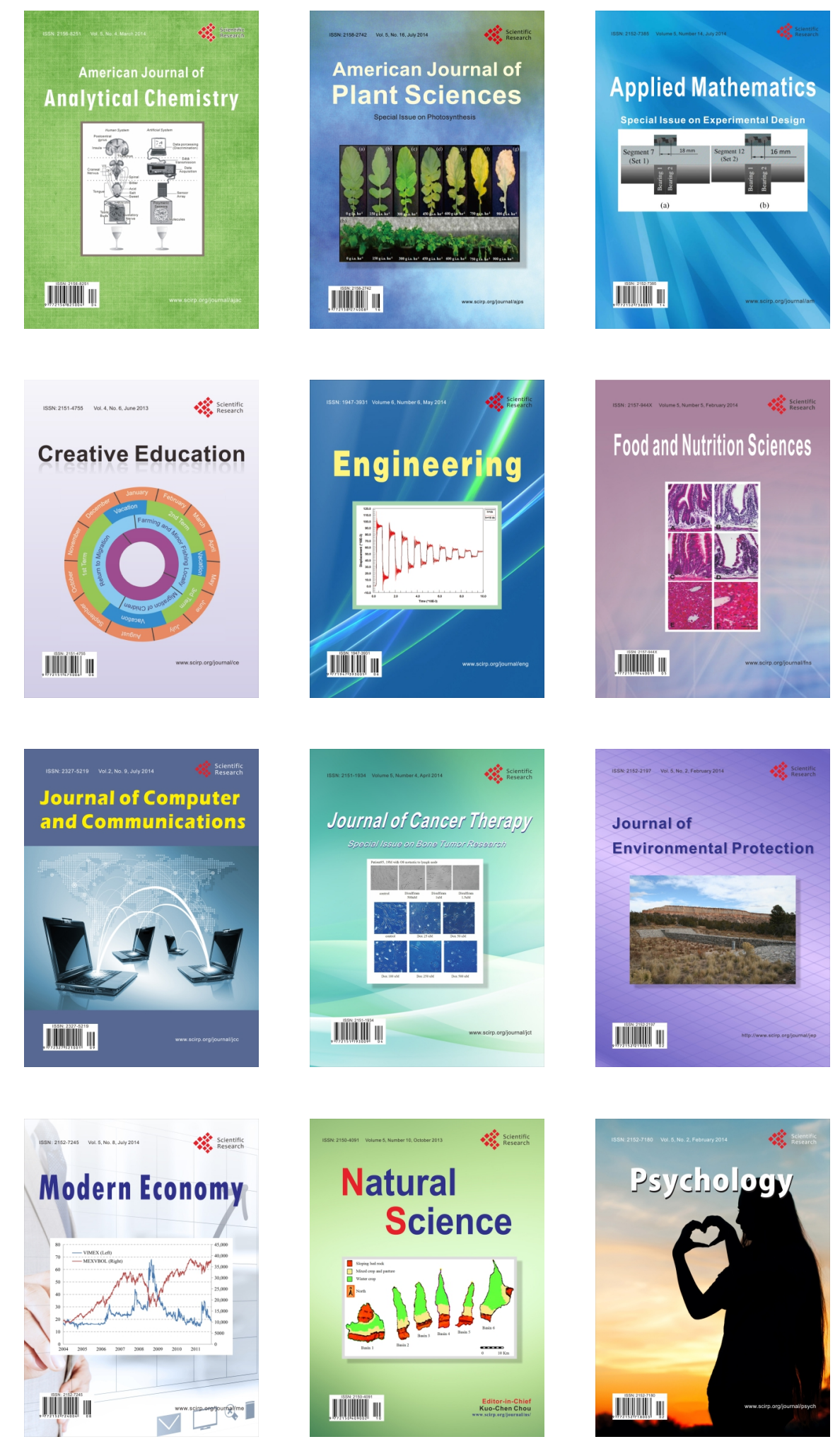